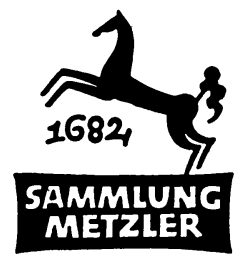

REALIEN ZUR LITERATUR

ABT.E:

POETIK 


\section{HEINZ GROTHE}

\section{Anekdote}

2., durchgesehene und

erweiterte Auflage

MCMLXXXIV

J. B. METZLERSCHEN VERLAGSBUCHHANDLUNG

STUTTGART 
Der Verfasser ist wie schon bei der ersten Auflage dieser Arbeit Herrn Bibliotheksdirektor Dr. Peter Liebenow von der »Amerika Gedenkbibliothek - Berliner Zentralbibliothek « für freundliche und hilfreiche Unterstützung zu besonderem Dank verpflichtet.

CIP-Kurztitelaufnahme der Deutschen Bibliothek

\section{Grothe, Heinz:}

Anekdote / Heinz Grothe. - 2., durchges. u. erw. Aufl. Stuttgart: Metzler, 1984.

(Sammlung Metzler; M 101: Abt. E, Poetik)

ISBN 978-3-476-12101-1

NE: GT

\section{110}

ISBN 978-3-476-12101-1

ISBN 978-3-476-04081-7 (eBook)

DOI 10.1007/978-3-476-04081-7

(C) 1984 Springer-Verlag GmbH Deutschland

Ursprünglich erschienen bei J. B. Metzlersche Verlagsbuchhundlung und Carl Ernst Poeschel Verlag GmbH in Stuttgart 1971/1984 
Vorbemerkung ................... 1

Einleitung .................... 2

I. Der Begriff $»$ Anekdote $\ldots \ldots \ldots \ldots \ldots \ldots \ldots \ldots 7$

a) Bedeutung und Entwicklung $\ldots \ldots \ldots \ldots \ldots \ldots$

b) Definition des Begriffs ................. 10

c) Bausteine zur Anekdotenbetrachtung . . . . . . . 19

II. Die Formen der Anekdote . . . . . . . . . . . 28

a) Die Urform: Die mündliche erzählte Anekdote . . . . 28

b) Die Kunstform: Die geschriebene Anekdote ..... 33

III. Die Grenzen der Anekdote . . . . . . . . . . . . . 36

a) Anekdote und Novelle . . . . . . . . . . . . . 38

b) Anekdote und Kurzgeschichte . . . . . . . . . . . . 42

c) Anekdote und Kalendergeschichte . . . . . . . 47

d) Anekdote und Schwank . . . . . . . . . . . . 51

e) Anekdote und Witz . . . . . . . . . . . . . 54

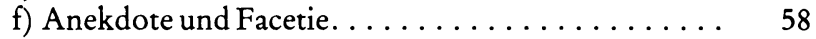

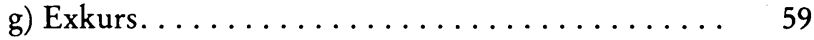

IV. Die Geschichte der Anekdote. . . . . . . . . . . 61

a) Die Anekdote im internationalen Bereich ....... 61

b) Die Anekdote im deutschsprachigen Raum . . . . . . 74

1. Die frühe Zeit bis zum 18. Jahrhundert $74-2$. Die klassische deutsche Anekdote bei Kleist und Hebel 78 3. Die Anekdote im 19. Jahrhundert - Karl Müchler und andere 86 - 4. Die Erneuerung der deutschen Anekdote Wilhelm Schäfer, Hans Franck, Josef Winckler, Wilhelm von Scholz, Sigismund von Radecki, Eugen Roth, Franz Carl Weiskopf, Friedrich Torberg $92-5$. Die Anekdote in der Nachfolge Wilhelm Schäfers, Karl Lerbs, N. O. Scarpi 110

V. Typengeschichte der Anekdote ............ 129

a) Die Klatschanekdote . . . . . . . . . . . . . . 129

b) Die Standesanekdote . . . . . . . . . . . . . 131

c) Die geschichtliche Anekdote. . . . . . . . . . 133

d) Die politische Anekdote . . . . . . . . . . . . . 135 
e) Die publizistische Anekdote . . . . . . . . . . . . 137

f) Die landschaftsgebundene Anekdote . . . . . . 139

g) Die Wanderanekdote . . . . . . . . . . . . . 143

VI. Dergesellschaftliche Aspekt. . . . . . . . . . . . 149

VII. Die Lebenskraft der Anekdote. . . . . . . . . . . 153

VIII. Anekdote und Didaktik . . . . . . . . . . . . . 157

Register...................... 165

\section{ABKÜRZUNGSVERZEICHNIS}

BLVS Bibliothek des Literarischen Vereins Stuttgart

Diss. Dissertation

dt.DT. deutsch, Deutsch

DU Der Deutschunterricht (Stuttgart)

DVjs. Dt. Vierteljahrsschrift für Literaturwissenschaft und Geistesgeschichte

Euph. Euphorion

Jb.Jbb. Jahrbuch, Jahrbücher

Jh. Jahrhundert

Mh. Monatshefte

RL Reallexikon der dt. Literaturgeschichte

Slg Sammlung

SWB Sachwörterbuch der Deutschkunde

ZfDk. Zeitschrift für Deutschkunde 
Die erweiterte Neufassung dieses Bandes bleibt ihrer Zielsetzung treu. Sie folgt auch nicht Anregungen, zu den mehr als drei Dutzend Definitionen der Anekdote eine eigene beizusteuern.

Es war notwendig, das inzwischen erheblich gewachsene Angebot literarischer und wissenschaftlicher neuer Arbeiten einzufügen. Besondere Aufmerksamkeit galt der Primär- und Sekundärliteratur der Anekdote. Über zweihundert Neuerscheinungen von Anekdotenbänden waren zu berücksichtigen. Eine Vielzahl theoretischer Untersuchungen in Dissertationen, Fachbroschüren, Aufsätzen in Jahrbüchern, Einleitungen zu Sammelbänden, in Zeitschriften, Wochen- und Tageszeitungen gesellten sich hinzu. Auch manche ältere Literatur zur >Anekdote $<$ wurde zusätzlich entdeckt.

Die $>$ Anekdote gibt sich nach wie vor als lebendiges Zeugnis der Literatur der jeweiligen Zeit. Das anhaltende Interesse bezeugt, daß ihre Entwicklung nicht überholt ist und daß sie sich (vermutlich) ihre >offene Form $<$ erhalten wird.

Seit über zweihundert Jahren hat sich die >Anekdote ihre Daseinsberechtigung bewahrt. Ihre Anfänge in Deutschland liegen im ,Vorfeld ‘ von Kleist und Hebel. Um die Jahrhundertwende bemühte man sich um eine Wiederbelebung der >Anekdote،. Während des Wechselspiels in den Jahrzehnten jüngerer Zeitrechnung hat offenbar die Anziehungskraft der >Anekdote « nicht nachgelassen.

Mit unserer Abhandlung wurde 1971 ein Anstoß zu intensiverer Beschäftigung mit der >Anekdote< gegeben; die nunmehr erweiterte Darstellung möchte einen Grund dazu legen, daß auf ihm in den "verschiedensten Richtungen und mit den verschiedensten Methoden weitergebaut werden kann" (wie es Bausinger in seinen "Formen der Volkspoesie « ausgedrückt hat).

Der Verfasser hat bei seiner Neufassung von vielen Seiten Anregungen erfahren. Dafür hat er zu danken. Besonderer Dank gilt Herrn Prof. Jürgen Hein (Münster/Köln). Den Arbeiten von Rudolf Schäfer, Rainer Schöwerling und Walter Ernst Schäfer fühlt er sich insgesamt verpflichtet. 